\title{
Imagining the Unimaginable: Narratives of the Big Bang \\ Time, Space, Matter, Energy
}

\author{
Barry Wood \\ University of Houston
}

Abstract: The term big bang has an uneasy history of problematic and misleading implications. As a derogatory and simplistic metaphor it is incompatible with current understandings of Planck time, inflationary theory, selforganizing dynamics, and emergent complexity. Scientific theory is judged and accepted as much by vocabulary as by content; content-specific nomenclature is a crucial key to understanding. Additionally, imaginative nomenclature that triggers a narrative meets the human need for a relevant story. Creative descriptions for the big bang that reformat it as a complementary cluster of stories are herein proposed with acronyms appropriate to the action of the big bang and meanings consistent with current science; approaches through analytical physics and complex mathematics are here replaced with series of suggestive narratives. Together, they combine to create a multi-strand narrative compatible with our present understanding of cosmic history understood as the Grand Sequence or the Big Story. Additionally the acronyms central to this presentation emphasize that the foundations of reality as we know it-Time, Space, Matter, and Energy — did not exist before the big bang but were in fact created in that event.

Anyone venturing into astronomy or cosmology inevitably has to grapple with the big bang. While there are more complex topics in science-quantum physics, for instance, or cell signaling — big bang cosmology challenges our customary experience and understanding of the world. It is simply impossible to imagine how an entire universe could unfold from next to nothing or how time and space - the apparent containers of everything we know-could have come into existence rather than always existing. Lawrence Krauss (2012) makes "a universe from nothing" seem simple, logical, and inevitable; most of us find it otherwise. The following notes provide a brief history of the big bang idea, its eventual acceptance, and current understandings organized into some unique ways to historicize or narrativize what we might more congenially call the big beginning or the first event.

\section{History of the Term Big Bang}

Marshall McLuhan, noted for his aphoristic analyses of communication, often said that to label is to libel. The term big bang, coined in a radio broadcast in 1949 by astronomer Fred Hoyle, provides an apt illustration: his off-the-cuff invention of the term was dismissive and derisive. "In the 1950s," Steven Weinberg has said, "the study of the early universe was widely regarded as not the sort of thing to which a respectable scientist would devote his time" $(1977,4)$, to which Alan Guth has added, "neither he [Hoyle] nor anyone else would use such a silly phrase [as "big bang"] in a dignified scientific publication" (1997, 99).

In the early twentieth century, the prevailing cosmology favored an eternal universe which seemed to fit contemporary understanding of the laws of physics, though a universe with no beginning is as conceptually difficult as one with a definite beginning. The replacement theory originated with observations in the 1920s that suggested the Universe may have had a beginning and thus a history. It began with Edwin Hubble's discovery that galaxies were retreating at varying velocities and calculations that distant galaxies were moving faster in a systematic fashion. Tracking them backwards in time suggested a common origin, though Hubble (1929) thought it "premature to discuss in detail the obvious 
consequences of the present results" (Bartusiak, 423-424). George Lemaitre (1931), however, felt no hesitation in putting forth the idea that the whole universe had been produced by the disintegration of a "primeval atom" (Bartusiak, 324). Arthur Eddington (1931) recognized the consistent and inescapable logic of an expanding universe with a distinct beginning in time, but resisted it: "Philosophically the notion of a beginning of the present order of Nature is repugnant to me. I should like to find a genuine loophole" (Bartusiak, 450). Preferring a steady-state, eternal Universe, Eddington argued that "the most satisfactory theory would be one which made the beginning not too unaesthetically abrupt. ... There is no hurry for anything to begin to happen" $(1932,56)$. This reticence continued for several decades. When George Gamow cautiously opened up his discussion of the creation of the universe, he avoided the term big bang, preferring instead to speak of "the great expansion" following an "originally homogenous primordial material" $(1952,4)$, implying a dramatic but quiet beginning. Even in his discussions of Hoyle's opposing concept of an eternal, steady-state universe, Gamow declined to dignify the big bang by even mentioning Hoyle's dismissive invention of the term. Resistance took many forms. Until his death in 2001, Hoyle maintained his opposition to the idea of a universe with a beginning despite the accumulating evidence in the latter half of the twentieth century.

Acceptance of this idea was delayed until the 1960s when evidence for the theory of the big bang began to accumulate. The first evidence arrived somewhat by accident in 1965. Two scientists working for Bell Laboratories in New Jersey were attempting to eliminate background noise in newly designed radio antennae. Arno Penzias and Robert Wilson were skilled technicians, not astrophysicists; thus, when they discovered that "noise" was arriving wherever they pointed their antennae, they decided consultation was in order. While the signal was exceedingly weak, its arrival from every point in the universe added up to a colossal amount of energy. When scientists from Princeton looked over their telescope, rechecked every aspect of its design, and were unable to eliminate this persistent noise, they concluded they were hearing something that had been predicted - if the big bang had actually occurred: leftover radiation emanating from every part of the universe, red shifted beyond the visible spectrum.

By the early 1980s, following this telltale evidence of what is now called the cosmic background radiation (CBR), the libelous intent of the term big bang had virtually disappeared: Weinberg (1977) had outlined "the first three minutes" of the universe with impressive mathematical support, Joseph Silk had published The Big Bang (1980), and Alan Guth (1981) had introduced his clarifying theory of superinflation occurring during a few trillionths of the first second. Notably, all three provided descriptions and analyses that far surpassed the limited connotations of Hoyle's "big bang." Since then, the term has continued to gain traction through various analyses and retellings (Trefil 1983; Gribbin 1986; Smoot 1993; Singh 2004).

Even after the event of the big bang had become accepted by the scientific community, the term itself remained bothersome, implying the universe began with an explosion. In an e-mail to this writer (11 November 2011) astronomer Eric Chaisson of Harvard University noted that he refers to "the $\mathrm{t}=$ 0 event" while Steven Weinberg tends to speak of "primordial chaos." In the 1980s Carl Sagan initiated lunchtime discussions at Harvard to find a better name and even ran a contest in his class, but struck out, while Alan Guth and Joseph Silk use "big bang" without apology. In the 1990s, Timothy Ferris, then editor of Sky and Telescope, called the big bang "a misleading, ugly and trivializing name." Accordingly, he announced a renaming contest that drew 13,099 entries - few from cosmologists, many from punsters, creative humor abounding — with judges Carl Sagan, Hugh Downs, and Ferris himself. The results announced in the February 1994 issue found 
none achieving what Sagan called the "felicity" of big bang. As Eric Chaisson has summarized the issue, Occam's razor seems to apply; big bang is simple, efficient, and dramatic or, as Simon Singh puts it, "short, punchy and memorable" (2004, 483).

The big bang metaphor, however, diminishes what quantum physics, mathematical reconstruction, and computer simulation indicate occurred at the inception of the universe, which is too easily visualized as a bursting balloon at a child's birthday party, or the detonation of a bomb. Supernova and kilonova explosions, recently observed in real time (Soderberg 2008; Cho 2017) continue to reinforce this version of events. Undoubtedly, explosion imagery is partly responsible for discomfort with and rejection of the entire idea. A communication challenge remains: undoing misconceptions inherent in the term and developing more accurate descriptions. The big bang is now understood as a complex event bracketed between timelessness and history, between nothingness and, eons later, an estimated 350 billion galaxies arranged in filaments across hundreds of millions of light years, a cosmology vast and unimaginable. Given the limiting features of all language, illustrative metaphors and narratives should harmonize with this new cosmology.

As humans situated within a limited spacetime matrix, we are naturally led to an idea of the big bang as occurring within Newtonian space and time. As such, the term implies an event in a material medium located in space at a point in time. Logically, however, there was no physical or temporal framework yet available; all attempts to locate this event require a space-time framework that unfolded later. Thus, in comparison with other uses of metaphor in astrophysics-white dwarfs, black holes, or dark energy - the big-bang metaphor distorts and evades the uniqueness of what we now believe happened.

A term such as big beginning (BB) is a close variant of big bang that entails fewer conceptual hazards but, while less problematic, it provides little more than an alternate name for a temporal (t) point: Chaisson's $t=0$ event. As an event with meaning for the present human situation, however, the big beginning must necessarily be imagined in narrative form, a first episode in what Chaisson (2006) calls the "epic of evolution," for narrative, as Donald Brown (1991) puts it, is a "human universal" such that young children and humans from every culture exhibit narrative understanding as a fundamental cognitive endowment. While early accounts of the big bang by Joseph Silk (1977) and Nigel Calder (1983) included elements of narrative, they also included minute dissections of the first microseconds into "eras" (Planck, Inflationary, Hadronic, Leptonic, and Decoupling), introducing a complex chronology mathematically precise but seemingly irrelevant and excessively detailed for anyone outside a very limited specialty. From the standpoint of quantum physics, such microscopic periodization may be mathematically precise and thus necessary, but ordinary learners, who make up more than $99 \%$ of the population, will prefer and in fact require some kind of meaningful narrative of how everything began, especially because "everything" includes their world, their possessions, their families and friends, and their own minds and bodies. Pure objectivity is a necessary ideal of scientific investigation and description, but relevance to self is the emotionally necessary foundation of interest and attention.

Assuming "big beginning" as a non-contentious synonym for big bang, the task of communication must be redefined: How can this incomprehensible event when time began, space unfolded, matter appeared, and energy bifurcated into various forces be formulated as imaginative narratives that will broaden and deepen its meaning and significance in harmony with discoveries over the past half century? How can we revision it in an interesting fashion, avoiding the complex physics which is best left for scientists, and capture the overall story? The following mini-narratives conveniently capture and 
summarize big-bang narratives this has writer has developed for a core-curriculum course that includes Cosmos, Earth, Life, Humanity, and Culture - a history of the universe formulated in a framework friendly to the humanities: Cosmic Narratives (Wood 2011 ; 2016). In literary terms, narratives can be as extended as Gustave Flaubert's three-hundred-page Madame Bovary or as brief as a simple declarative summary: "Romantic fantasies destroyed Emma Bovary." News anchors regularly introduce the news hour with a series of brief sentences that will be expanded to stories of several minutes later in the broadcast. Here we are looking for succinct summary narratives of the big bang that are capable of expansion into a detailed and comprehensive story. For interest, we have looked for stories that could be summarized in acronyms. Our current understanding of the big bang includes the creation, not simply of the Universe, but the underlying structures of reality_-Time and Space, Matter, and Energy — which are necessary for our existence. To link the story of the big bang to these underlying structures, we have imaginatively used TIME, SPACE, MATTER and ENERGY as acronyms. This presentation should not be regarded as a conceptual change, new science, or alternative history but rather as imaginative ways of recasting science and history for a new view of the origin of things.

\section{The Initial Moment of Emergence (TIME)}

Without change, there is no way to measure time. Our present understanding indicates that there was no change before the event of the big beginning and therefore no time; the big bang marks the beginning of change and time. An expanded version of the big beginning, The Initial Moment of Emergence (TIME), signifies the beginning of temporality as we know it along with the concept of emergence basic to cosmic history. TIME thus provides a useful summary that is pedagogically useful: once it is introduced it tends to stick. I find students adopting it in written work in place of the traditional term "big bang."

In thinking about the beginning of the universe (or the world), we tend to wonder when it occurred, "it" being a particular datable point on a vast linear time scale. As Dalrymple (1991), Gorst (2001), and Montgomery (2012) have documented, numerous Medieval scholars made attempts to provide a date for the beginning of the world based on a literal reading of genealogies in the Bible, the only ancient source that appeared to cover the entire history of the world. Such historical constructions derived from an ancient book (biblios) might be called bibliotemporality. The second-century theologian, Theophilus of Antioch, provided the first, but at least 125 dates were calculated over the centuries. Eventually Bishop James Ussher's date of 4004 BC published in Annals of the World (1649) won acceptance, largely because of his close association with the British monarchy and thus the Church of England; his dates were printed in the margins of the King James Bible well into the twentieth century. Bibliotemporality has now been superseded by radiometric dating which has reset the beginning of the world at 4.5 billion years ago. Moreover, observations of a systematically expanding universe, the predicted discovery of the cosmic background radiation (CBR), and recent detection of vast gravity waves in the CBR have established and verified a cosmology in which the beginning of the universe itself is placed 9.3 billion years earlier- 13.8 billion years ago. Despite an inconsequential minority of dissenters, big-bang cosmology has won the day throughout the scientific community.

It must be admitted, however, that the big bang label is imaginatively and cognitively limited. It implies a massive explosion with flying debris in all directions. We are overloaded with explosions in movies, on the battlefield, and in increasing acts of terrorism, but explosions are destructive events whereas The Initial Moment of Emergence was a creative event; it provided the impetus for the formation of an immense collection of galaxies and 
planets that followed, and for the eventual rise of life that occurred on at least one planet billions of years later. In order to link the big beginning with the present universe, we need a narrative that unfolds its temporal stages (epochs, or eras). Additionally, we need essential punctuation pauses within the temporal narrative, for time, as Einstein theorized and science has since verified, is subject to velocity: hence the "atemporality" of light and other radiation recognized by Julius Thomas Fraser (1982) that preserves and delivers a record of all earlier times to the present. Other punctuation points include the "petrotemporality" of rocks and meteors that preserves past "times" of the 4.5-billion year history of Earth, and the "genotemporality" that records landmarks in the history of life within the human genome (Wood 2015b).

As a simple alternative for the big beginning, The Initial Moment of Emergence (TIME) provides a framework for such a narrative: it implies and confirms a basic assumption that an initializing event occurred at $\mathrm{t}=0$, before which no time existed, and follows this with a temporally organized process of emergence. All narratives begin with change occurring because of an initial disequilibrium, followed by a sequential ordering of events (Sacks1964; Bal 1985), a narrative pattern that gratifies a basic human need to understand causation (Abbott 2008, 41) — how and why things happened the way they did. TIME defines a change in time: emergence is a temporal process of "rising out" (Latin: e-mergere), a coming forth of something formerly hidden, and in fact is now seen as basic to modern cosmology - a process repeated through what Fred Spier (1996) calls the "domains" of cosmic, planetary, and human history. Mario Bunge $(2003,12)$ provides a succinct definition: "Wholes possess properties that their parts lack. Such global properties are said to be emergent." Emergence associated with new thresholds of complexity occurs throughout cosmic history; a 13.8-billion-year sequence of emergences is traceable through particulate, stellar, chemical, biological, anthropological, and cultural development, thus casting all of cosmic history as evolutionary-a series of emergences associated with new layers of tiered complexity.

Emergence as the narrative thread of evolutionary and human history has been recognized over several decades by Norman Berrill (1955), Alan Broms (1961), John Pfeiffer $(1969,1977)$, Charles Maisels (1990), and Steven Johnson (2001); additionally, Mark Bedau and Paul Humphreys (2008) have gathered perspectives from more than twenty philosophers and scientists. Emergence is thus one of the most intensively studied aspects of cosmic history, perhaps because it is quietly recognized as a replacement for the millennia-old mythology of supernatural design as the organizing principle of the material Universe. Emergence describes the appearance of four forces from a single undifferentiated energy and the subsequent emergence of quarks that make up the various particles of elemental matter. Emergence captures the appearance of new properties in each of the elements fused from hydrogen in stellar furnaces, supernova, and kilonova explosions. At the chemical level, emergence describes the appearance of new properties when elements are combined to create millions of familiar compounds and, later, the achievement of reproductive capacity when complex arrangements of chemical molecules form living cells. Laboratory scientists seem reticent to dwell on these rather astonishing results of emergence, perhaps apprehensive that theology or mysticism may make inroads into science. But we should note that emergence even at the level of the material world is a mysterious process; in fact, the astonishing capacity for creativity in the material world has periodically given rise to connotations of spirituality, as in the work of Edmund Sinnott (1955), Pierre Teilhard de Chardin (1959), and Ursula Goodenough (1998). However, emergence remains a neutrally safe concept implying an appearance of 
innovation and complexity without prior causation. Emergence is also central to human organization as we witness the innovation and creativity emerging from social and cultural complexity and remarkable social applications emerging from the mechanical complexity of computers and the Internet. The Initial Moment of Emergence gives birth to Time, the thread upon which all subsequent emergences and complexities are strung in the Grand Sequence of cosmic history.

\section{Single Point Achieving Cosmic Extension (SPACE)}

Two spatial descriptions of the big beginning emphasize cosmic history as narrative: Single Point Achieving Cosmic Extension, and Singularity Potential Activating Cosmic Expansion (SPACE). Both add additional plot strands in the unfolding narrative following TIME. The former, focusing on the word "achieving," emphasizes a final spatial result; the latter, utilizing the word "activating," links tidily with the "initial moment" of TIME. Both introduce connotations at the boundaries of imagination, but explanation makes them cognitively accessible. "Achieving" and "activating" imply change in time and thus distinctive narratives. According to our present understanding, no spatial extension existed before TIME. "Single point" and "cosmic extension" provide a dramatic contrast between an infinitesimal, metaphorical "space" at $t=0$ and the nearly infinite expanse of real space eventually achieved; the visible universe now measures 26 billion light years in diameter, though its full extent is much greater. "Singularity potential" and "cosmic expansion" focus attention on a different aspect of origins and effects. Activation began at a point, though not a point "in" space understood as a container of things and events; rather, this event occurred at all points or, conversely, all points began as one point prior to the unfolding of space. Indirectly, the notion of "one point prior to the unfolding of space" sets aside the imaginative difficulties that virtually everyone puzzles over: How big is space? Where does it end? What lies beyond the end of space? All such questions are unanswerable because they are framed within a Newtonian space which did not yet exist. The creation of space by expansion renders old questions obsolete and makes tenable the logic of "a universe from nothing" described by Lawrence Krauss (2012). The initial point from which all points in space emerged, understood as devoid of physical dimension or position, is termed a singularity, although it encompasses the capacity for infinite multiplicity. An additional emphasis of this acronym is the introduction of expansion as now understood, and its corollary, inflation, part of big-bang theory ever since Guth (1981) proposed it and now apparently verified by polarization within the CBR. SPACE describes the initial event in terms of extension - not the expansion of matter "into" space but as the expansion of space itself between galaxies and galactic clusters, a recognition that requires the theory of dark energy to account for observations otherwise unexplainable. The value of SPACE is its incorporation of concepts on the leading edge of our present scientific understanding.

\section{Matrix Acting Toward Titanic Exothermic Radiation (MATTER)}

With the introduction of a third acronym, we begin to sense the depths of creativity implied in the big beginning as we now understand it. Matrix Acting Toward Titanic Exothermic Radiation (MATTER) carries a rich set of narrative connotations.

Archaically, matrix refers to the womb, a meaning tracing to the Latin mater (mother) and numerous cognates in Indo-European languages. Additionally, matrix provides a context where properties yet to emerge are implicit, folded in (im-plicatus) until they become explicit, or unfolded. In modern usage, a matrix encloses while ultimately imparting an emergent form or shape that develops and evolves with unwrapping (de-volupere) or turning outwards 
(e-volvere). The result is a titanic rush of energy, heat, and radiation akin to the torrential rain of a typhoon. Titanic derives from the Titans of Greek mythology, a race of giants who, among other cosmic disruptions, raised Chronos (time) and Typhon (storms) to rule the Universe - analogous perhaps to contrasting forces of order and entropy.

The radiation component of MATTER actually precedes matter as we know it, referring to a limited period following the big beginning of approximately 300 to 400 thousand years when the initial undifferentiated plasma was so dense, entangled, and constrained that radiation could not escape, thus rendering it invisible today. This constraint is explained by quantum physics: energy is packaged as discreet quanta, which for a few hundred thousand years were packed too densely to escape. But energy as discrete quanta underlies many features of the universe scientists have now discovered: the exchangeability of matter and energy, the regular succession of atomic mass in the chemical elements, consistent spectrographic signatures for elements and compounds, and measurable isotopic decay utilized in radiometric dating. "Exothermic radiation" describes the torrential outpouring of heat attending the earliest minutes following the event describe as TIME and SPACE. As a possible alternative for titanic, torrential is equally connotative since its Latinate meaning is scorching or boiling (torrens).

While MATTER refers to this limited era when the material universe emerged, the unspoken connotations of the acronym suggest a far broader narrative encompassing the entire material universe as we know it today that has descended and evolved from the exothermic radiation from the initial event.

\section{Entropic Nexus Emitting Radiation, Galaxies, and You (ENERGY)}

A fourth narrative for the big beginning may be summarized as Entropic Nexus Emitting Radiation, Galaxies, and You (ENERGY), a formulation that carries the narrative far beyond radiation, the final chapter implied by the R of MATTER. In its inclusion of both entropy and you, this phrasing defines divergent cosmic trends: the paradox of a universe tending toward both disorder in its initial dispersal of energy and localized order and pattern described as "sensitive chaos" by Theodor Schwenk (1965). Subsequently, order has been explored in tandem with complexity emerging from multilevel self-organizing dynamics formulated by Erich Jantsch (1980), Ilya Prigogine (1984), Stuart Kauffman (1995), and Philip Ball (1999). At one extreme, the second law of thermodynamics governs a dissipating universe where accelerating expansion seems to foreshadow an irrecoverable scattering of matter and energy at some distant point, perhaps 100 billion years in the future. The earliest sign of an entropic universe was detected when the Cosmic Background Explorer (COBE) recorded the cosmic background radiation (CBR) - a nearly uniform mist of pure radiation later measured by the Wilkinson Microwave Anisotropy Probe (WMAP) at less than three degrees above absolute zero with variations across the entire universe of no more than a few thousandths of a degree. If we had no knowledge of subsequent cosmic history with the CBR as our only clue, we might conclude that the vast creative energy of an expanding universe had been utterly lost to the implacable power of entropy within 400,000 years after TIME.

The first observational proof of order emerging within a larger entropic context appeared when telescopes were focused on middle distances a billion or two light years after TIME. Here miniscule variations in temperature corresponding to equally miniscule variations in density in the CBR were recognized as forerunners of galaxies and chains of clustered galaxies spread like jeweled filaments across vast regions of space. Utilizing the enormous power of the Hubble Telescope combined with microlensing, we have extended our view back in time more than 13 billion light years, discovering billions of stars clustered in fledgling galaxies within 
half a billion years after the big beginning. Our own Milky Way and most other galaxies appear to have formed early in the history of the universe, 10 to 13 billion years ago. Since then they have continued to draw in matter from their own region with larger galaxies occasionally swallowing smaller ones that venture too close. In general, though, galaxies are the longest-persisting entities in the universe, except for atoms. Their longevity makes them the most significant entity emerging from the original cloud of elemental dust to their status as host for our own existence. Galactic organization is minimallimited to the fine balance between the momentum of orbiting stars, an enormous amount of invisible dark matter, and the universal force of gravity that keeps hundreds of billions to a trillion stars together. The energy of momentum and the force of gravity working together maintain the order of galaxies as continuous with and emerging from the original nexus of matter-and-energy.

While many of the earliest galaxies were globular with little evident order beyond clustering, the most evolved galaxies have attained the visible symmetry of a vast flattened disc. As non-entropic concentrations of matter and energy, galaxies play host to stellar nurseries and billions of stars. The organization and structure of galaxies is relevant once we consider the range and variety of stars they engender. A galaxy provides a variety of stellar environments, from the frantic chaos of inner regions to the quieter realms of far flung galactic arms. In the inner regions stars orbit the galactic center at enormous speeds; their orbits are random, with interference and collisions more likely. In the outer regions of the large disc-shaped galaxies, stars run in roughly parallel paths, thus providing undisturbed stability that may last for billions of years. Stellar nurseries are evident through most galaxies but those of importance for higher levels organization occur in the central to outer regions of galaxies where the new stars that emerge have time and space to evolve more complex kinds of order.
The mathematics of stellar evolution is complex (Harpaz 1994; McWilliam 2004) but the story is simple (Gribbin 2000; Chown 2001), and it is a story that leads to humans and other life that may inhabit the universe as its final chapter. Any star significantly larger than the sun is subject to an enormous gravitational load; increasing density and rising temperature at its core turn it into a cosmic furnace that cooks the most basic element, hydrogen, into the next twenty-five. These and a score of others above iron on the Periodic Table are created and scattered through space during violent supernovas, and our most recent observations suggest that the upper forty or so, including gold, platinum, and uranium are fused in collisions of neutron stars (Cho 2017; Bloom 2017); these are perhaps a thousand times more violent and thus are known as kilonovas (kilo: thousand). The array of ninety-two sequentially constructed elements found in supernovae and kilonovae debris signals the emergence of complex chemical order from apparent chaos. Subsequently, within select regions near second-, third-, or fourthgeneration stars, an additional reversal of entropy occurs - a counter tendency where higher elements provide material for new compounds, with some acting as catalysts in the creative complexity of the rest. Left-over debris from star formation accretes to form a variety of planets, some rocky that become pockets of stability where, in at least one instance, matter and constant energy flow gave rise to progressive forms of emergent complexity: lifeself-replicating entities populating every ecological niche of the planet. In time, the fundamental sensitivity of such entities led to millions of species, aggregate communities, culture, thought, and imagination. ENERGY captures this force of counterentropy in its description of an "entropic nexus emitting radiation, galaxies, and you."

The inclusion of You shifts the emphasis of TIME, SPACE and MATTER in a radically new direction, which is unapologetically subjective; indeed, anthropocentric. More than half a century ago, the 
Canadian microbiologist Norman Berrill wrote a book called You and the Universe (1958). Published a year after the now-famous paper, "Synthesis of the Elements in the Stars" (Burbidge et al 1957), Berrill's presentation made clear for the first time that cosmic history unfolds as a continuous narrative from stardust (hydrogen, carbon, nitrogen, and oxygen, etc.) to the complex cells of which we humans are made. The universe displays multitudinous histories and entities, some of which - brown dwarfs, trilobites, dinosaurs, Neanderthals - have ended with extinction, but life has bifurcated into 300 million separate species, ninety-nine percent of which are now extinct. The three million alive today represent no more than one percent of all that have ever lived - though this number is still too great for any biologist to understand. But Berrill was interested in the specific pathway that provides a cosmic genealogy for humanity. Subsequent writersPreston Cloud (1978), Joel Primack (2006), Cynthia Stokes Brown (2007), Brian Swimme (2011), and John Hands (2017) - have restated his theme with varying creative emphases. Tyler Volk (2017) has introduced the term "combogenesis" to describe the new foundation for a creative beginning that occurs with each successive emergence - "from quarks to culture" - that he calls the "grand sequence." An interesting narrative innovation has the Universe telling its own story (Darling 1989), the first of a trend towards an autobiographical history of life on Earth (Ridley 2000; Dawkins 2004); here cosmic evolution is cast in a framework of biological memoir. We live within what Brian Swimme called "the universe story" (1992) and Gianluca Bocchi termed "the narrative universe" (2002).

As synthesizers of scientific ideas, some contemporary writers have sought to make advanced science of our time accessible for the non-scientific reader by providing narrative bridges between the "two cultures" (Snow 1960), the sciences and the humanities (Wood 2013). Carl Sagan (1980) achieved early visibility and brought cosmology out of the shadows; Neil de Grasse Tyson (2004) has achieved similar success. Jennifer Morgan (2002, 2003, 2006) has created a beautifully illustrated, three-volume version of the universe story for children. Expanding on the idea of "cosmic education" outlined by Mario Montessori (1976),Michael and D’Neil Duffy (2014) have articulated five "great stories" for the elementary classroom. A sequence of "big story narratives" (Wood, 2015a) for elementary and middle school children provides an alternate methodology. The presentation of the big beginning as a narrative of energy transformation that links its earliest events with our present human situation is perhaps the most potent way for cosmic history to achieve meaning and significance outside the specialized domains of science.

The appeal of reformatting the big beginning as TIME, SPACE, MATTER and ENERGY is both practical and pedagogical, an exercise in imaginative story making. Big History teachers who are not scientists tend to slide past the big bang, which is only fully understood by science specialists. Historians treat the history of civilization at length. Biologists emphasize evolution; geologists focus on the forces of plate tectonics and orogeny. But the big bang should not remain out of reach, an arcane arena of quantum physics. Properly understood, it is seen as laying down the foundation for everything that follows. College and university teachers bear responsibility for communicating the most advanced concepts from the sciences in terms not only comprehensible but also meaningful for the next generation. An analysis of the first microseconds into a series of fleeting "eras" registers the analytic power of advanced mathematics and quantum physics, but it loses traction for students whose primary interest lies in the humanities - or even for students in other branches of science. Given the fundamental allure of a meaningful story, the big beginning requires narrative connectivity with Earth, life, and the human situation. Above all, it needs imaginative presentation. 
The goal of the International Big History Association (IBHA) is to unify scientific knowledge in a grand framework defined as an "attempt to understand, in a unified, interdisciplinary way, the history of Cosmos, Earth, Life, and Humanity" (Christian 2011, 20). Norman Berrill (1958) and Preston Cloud (1978) produced impressive cosmic histories, though too early to include the opening big-bang chapter; more recent studies by David Christian (2004), Cynthia Stokes Brown (2007), Brian Swimme (2013), and Walter Alvarez (2017) — all prominent big historians - necessarily include it, though, as the title of this article implies, thinking about the big bang is always a matter of imagining the unimaginable. Tyler Volk (2017) has made the big-bang pre-atomic story of quarks as understandable as it may ever become for the non-scientist. Our series of narratives summarized as TIME, SPACE, MATTER, and ENERGY are not intended as scientific contributions but rather accessible literary versions of the big beginningan opening chapter and an evolutionary story that Darwin never dreamed of. At the same time, these acronyms double as the fundamental realities upon which the Grand Sequence of cosmic history has been written. Edward O. Wilson (1998) captured this sequence in the phrase "epic of evolution"; Eric Chaisson (2006) developed it into a narrative of seven ages; Cheryl Genet (2009) has assembled the conference proceedings of more than thirty scholars exploring "the evolutionary epic." Narrative as a fundamental method for presenting cosmology has earned a unique place straddling the sciences and humanities. This expansion of the human past - from history to big history - was first defended by David Christian (1991); it has gained acceptance in the new millennium, not only with the 2010 formation of IBHA but also affiliates in Europe and Asia. In the inaugural volume from the Santa Fe Institute dedicated to complexity science, David Krakauer et al (2017) have undertaken a sweeping examination of big-history theoretical foundations with essays from a wide range of scientists and social scientists. The Big History emphasis on the narrative continuity of cosmic history links every later development in the universe to the big beginning. The unity of space and time as worked out by Albert Einstein has gradually gained clarity. The interchangeability of matter and energy is less obvious; we tend to regard rocks, trees, insects, and people as made of different "stuff." Yet the material and movement of our bodies - every molecule of brain and bone, every heartbeat, every thought flickering across the cortex-derives from the history of the universe, tracing back through numerous energy transformations to exfoliating radiation when everything began.

New descriptions for the big bang or the big beginning, with its most recent emergent identified as you, emphasize the significance of humanity as the most complex product of the 13.8-billion-year history of the Universe. We may soon detect signs of life in nearby exoplanets, but confirmation that it has achieved complexity equal or greater than ours will require technological sophistication and communication that may lie decades or centuries in the future. For now, the miniscule 200-thousand-year history of Homo sapiens remains the final chapter in the narrative. In this light, it is appropriate to explore and reinvent our presentation of the big bang in order to set aside lingering misconceptions, explore its multiple story lines, and bring into focus the profound importance of this event as the departure point for a scientifically-based narrative leading to our own recent emergence.

\section{References}

Abbott, H. Porter (2008): The Cambridge

Introduction to Narrative. Second Edition.

Cambridge. University Press.

Alvarez, Walter (2017): A Most Improbable Journey: A Big History of Our Planet and Ourselves. New York: W. W. Norton.

Bal, Mieke (1985): Narratology: Introduction to the Theory of Narrative. Toronto: University of 
Toronto Press.

Ball, Philip (1999): The Self-Made Tapestry:

Pattern Formation in Nature. New York: Oxford University Press.

Bartusiak, Marcia, ed. (2004): Archives of the

Universe: A Treasury of Astronomy's Historic Works of Discovery. New York: Pantheon Books.

Bedau, Mark A. and Paul Humphreys, eds. (2008): Emergence: Contemporary Readings in Philosophy and Science. Cambridge, Massachusetts: MIT Press.

Berrill, Norman J. (1955): Man's Emerging Mind. New York: Dodd, Mead \& Company.

Berrill, Norman J. (1958): You and the Universe. New York: Dodd, Mead \& Company.

Bloom, Joshua S. and Steinn Sigurdsson (2017):

"A Cosmic Multimessenger Gold Rush." Science 358, 20 October, 301-302.

Bocchi, Gianluca and Mauro Ceruti (2002): The Narrative Universe. Trans. Luca Pelligrini, and Alfonso Montuori. Cresshill, New Jersey: Hampton Press.

Broms, Allan (1963): Our Emerging Universe. New York: Muller.

Brown, Donald (1991): Human Universals. New York: McGraw Hill.

Brown, Cynthia Stokes (2007): Big History: From the Big Bang to the Present. New York: The New Press.

Bunge, Mario (2003): Emergency and Convergence: Qualitative Novelty and the Unity of Knowledge. Toronto: University of Toronto Press.

Burbidge, Eleanor M. et al (1957): "Synthesis of the Elements in the Stars," Physical Review 20, No. 4, pp. 547-650.

Calder, Nigel (1983): Timescale: An Atlas of the Fourth Dimension. New York: Viking Press.

Chaisson, Eric (2006): Epic of Evolution: Seven Ages of the Cosmos. New York: Columbia University Press.

Cho, Adrian (2017): “A Spacetime Tremor and a Celestial Light Show." Science 358, 20 October, 282283.
Chown, Marcus (2001): The Magic Furnace: The Search for the Origin of Atoms. New York: Oxford University Press.

Christian, David (1991): "The Case for 'Big History'." The Journal of World History 2, No. 2, pp. 223-238.

Christian, David (2004): Maps of Time: An Introduction to Big History. Berkeley: University of California Press.

Christian, David (2011): "The Evolution of Big History.” A Big History Perspective. Eds. L. E. Grinin, A. V. Korotayev, B. H. Rodrigue. Volgograd: 'Uchitel'. pp. 20-25.

Cloud, Preston (1978): Cosmos, Earth and Man: A Short History of the Universe. New Haven: Yale University Press.

Dalrymple, G. Brent (1991): The Age of the Earth. Stanford: Stanford University Press.

Darling, David (1989): Deep Time: The Journey of a Single Sub-atomic Particle from the Moment of Creation to the Death of the Universe-and Beyond. New York: Bantam.

Dawkins, Richard (2004): The Ancestor's Tale: A Pilgrimage to the Dawn of Evolution. New York: Houghton Mifflin.

Duffy, Michael and D'Neil Duffy (2014): Children of the Universe: Cosmic Education in the Montessori Elementary Classroom. New York: Montessori Services.

Eddington, Arthur (1931): "The End of the World: From the Standpoint of Mathematical Physics," Nature 127, pp. 447-453.

Eddington, Arthur (1932): The Expanding Universe. Cambridge: Cambridge University Press.

Fraser, Julius Thomas (1982): The Genesis and Evolution of Time. Amherst: University of Massachusetts Press.

Gamow, George (1952): The Creation of the Universe. New York: Viking Press.

Genet, Cheryl et al (2009) (Eds): The Evolutionary Epic: Science's Story and Humanity's Response. Santa Margarita, California: Collins Foundation 
Press.

Goodenough, Ursula (1998): The Sacred Depths of Nature. New York: Oxford University Press.

Gorst, Martin (2001): Measuring Eternity: The

Search for the Beginning of Time. New York:

Broadway Books.

Gribbin, John (1986): In Search of the Big Bang:

Quantum Physics and Cosmology. New York:

Bantam.

Gribbin, John (2000): Stardust: Supernovae and

Life - the Cosmic Connection. New Haven: Yale University Press.

Guth, Alan H. (1981): "The Inflationary Universe:

A Possible Solution to the Horizon and Flatness

Problems," Physical Review D 23, pp. 347-356.

Guth, Alan H. (1997): The Inflationary Universe:

The Quest for a New Theory of Cosmic Origins.

Reading, MA: Addison-Wesley.

Hands, John (2017): CosmoSapiens: Human

Evolution from the Origin of the Universe. New

York: Overlook Duckworth.

Harpaz, Amos (1994): Stellar Evolution.

Wellesley, Massachusetts: A. K. Peters.

Hubble, Edwin (1929): “A Relation between

Distance and Radial Velocity among Extra Galactic

Nebulae," Proceedings of the National Academy of

Sciences, 15. In Bartusiak, pp. 421-424.

Jantsch, Erich (1980): The Self-Organizing

Universe: Scientific and Human Implications of

the Emerging Paradigm of Evolution. New York:

Pergamon Press.

Johnson, Steven (2001): Emergence: The

Connected Lives of Ants, Brains, Cities, and

Software. New York: Simon and Schuster.

Kauffman, Stuart (1995): At Home in the Universe:

The Search for the Laws of Self-organization and

Complexity. New York: Oxford University Press.

Krakauer, David, et al, eds. (2017). History, Big

History \& Metahistory. Santa Fe: SFI Press.

Krauss, Lawrence M. (2012): A Universe from

Nothing: Why There is Something rather than

Nothing. New York: Free Press.
Lemaitre, Georges (1931): "The Beginnings of the World from the Point of View of Quantum Theory," Nature 128. In Bartusiak, pp. 323-324.

Maisels, Charles K. (1990): The Emergence of Civilization: From Hunting and Gathering to Agriculture, Cities, and the State in the Near East. London: Routledge.

McWilliam, Andrew et al, eds. (2004): Origin and Evolution of the Elements. Cambridge: Cambridge University Press.

Montessori, Mario (1976): Cosmic Education. Amsterdam: Association Montessori Internationale.

Montgomery, David R. (2012): The Rocks Don't Lie. New York: W. W. Norton.

Morgan, Jennifer (2002): Born with a Bang: The

Universe Tells Our Cosmic Story. Nevada City,

California: Dawn Publications.

Morgan, Jennifer (2003): From Lava to Life: The

Universe Tells Our Earth Story. Nevada City,

California: Dawn Publications.

Morgan, Jennifer (2006): Mammals Who Morph:

The Universe Tells the Evolution Story. Nevada City,

California: Dawn Publications.

Pfeiffer, John E. (1969): The Emergence of Man.

New York: Harper and Row.

Pfeiffer, John E. (1977): The Emergence of

Society: A Prehistory of the Establishment. New

York: McGraw Hill.

Prigogine, Ilya, et al (1984): Order out of Chaos:

Man's New Dialogue with Nature. New York:

Bantam.

Primack, Joel R. and Nancy Ellen Abrams (2006): The View from the Center of the Universe:

Discovering Our Extraordinary Place in the Cosmos. New York: Riverhead Books.

Sacks, Sheldon (1964): Fiction and the Shape of Belief: A Study of Henry Fielding, with Glances at Swift, Johnson and Richardson. Berkeley: University of California Press.

Sagan, Carl (1980): Cosmos. New York: Random House. 
Schwenk, Theodor (1965): Sensitive Chaos:

The Creation of Flowing Forms in Water and Air.

London: Rudolf Steiner.

Silk, Joseph (1988): The Big Bang. Revised and updated edition. New York: W. H. Freeman.

Singh, Simon (2004): Big Bang: The Origin of the Universe. New York: HarperCollins.

Sinnott, Edmund W. (1955): The Biology of the Spirit. New York: Viking.

Smoot, George, et al, eds, (1993): Wrinkles in Time: Witness to the Birth of the Universe. New York: Little Brown.

Snow, Charles P. (1960): The Two Cultures.

Cambridge: Cambridge University Press.

Soderberg, Alicia M., et al. (2008): “An

Extremely Luminous X-ray Outburst at the Birth of a

Supernova." Nature 453 (7194): 469-474.

Spier, Fred (1996): The Structure of Big History:

From the Big Bang until Today. Amsterdam:

Amsterdam University Press.

Swimme, Brian and Thomas Berry (1992): The

Universe Story: From the Primordial Flaring Forth to the Ecozoic Era-A Celebration of the Unfolding

of the Cosmos. San Francisco: HarperCollins.

Swimme, Brian T. and Mary E. Tucker (2011):

Journey of the Universe. New Haven: Yale

University Press.

Teilhard de Chardin, Pierre (1959): The

Phenomenon of Man. London/ New York: Collins.

Trefil, James S. (1983): The Moment of Creation: Big Bang Physics from Before the First Millisecond to the Present Universe. Mineola, New York.

Tyson, Neil de Grasse and Donald Goldsmith (2004): Origins: Fourteen Billion Years of Cosmic Evolution. New York: W. W. Norton.

Volk, Tyler (2017): Quarks to Culture: How We Came to Be. New York: Columbia University Press.
Weinberg, Steven (1977): The First Three Minutes: A Modern View of the Origin of the Universe. New York: Basic Books.

Wilson, Edward O. (1998): Consilience: The Unity of Knowledge. New York: Alfred A. Knopf.

Wood, Barry (2011): "Cosmic Narratives Course Launched," IBHA Newsletter, Vol. 1, No. 4.

Wood, Barry (2013): "Bridging the "Two Cultures': The Humanities, Sciences, and the Grand Narrative," The International Journal of Humanities Education 10, pp. 44-55.

Wood, Barry (2015a): "Big Story Narratives: Reframing K-12 Science Education," Proceedings: 13th Annual Hawaii International Conference on Education (HICE), January 5-8, 2015. Honolulu. pp. 1966-1991.

Wood, Barry (2015b): "Underlying Temporalities of Big History," KronoScope: Journal for the Study of Time 15, pp. 157-178.

Wood, Barry (2016): "Big History, Big Science, and Cosmic Narratives: Variant Approaches to the Big Story," From Big Bang to Galactic Civilizations: A Big History Anthology. 3 vols. Eds. Barry Rodrigue et al. Delhi: Primus. Vol. 2, 81-90. 
